\title{
Observational study of proximal fixation nail anti rotation and gamma nail in trochanteric fracture of Femur
}

\author{
Mohammad Sahamim ${ }^{1}$, Mohd Mohasin khan ${ }^{1}$, Cui Xuewen ${ }^{1}$, Harish singh ${ }^{2}$, \\ Pankaj kumar ${ }^{3}$ \\ ${ }^{, 1,2,3}$ Orthopedics Department No. 3, Affiliated Hospital of Jiangsu University, Zhenjiang, Jiangsu Province, P. R. \\ China
}

\begin{abstract}
The basic purpose of study is to find out advantage and disadvantage of fixation nail anti rotation and gamma nail in trochanteric fracture of femur. I observed about 100 cases of unstable fracture of femur in my hospital which was treated by anti rotational fixation nail of two years and took some data of gamma nail .I did observe that in PFNA blood loss was very less and duration of surgery was short and PFNA is very good in osteoporotic patients in compare to the gamma nail 3. Reduction of the fracture was considered good in both cases and radio graphically both device position was good postoperatively. Intra operative complication was comparatively more in gamma nail than anti rotational fixation nail such as shaft fracture of the femur, blood loss, duration of surgery. Post operative complication was not seen in both cases such as nonunion, malunion.so after studying both cases I did not found so much difference between these two devices which are used in trochanteric fracture by the orthopedic surgeons but very few advantage with the proximal fixation nail. So the both intrameddulary nailing device was good compare to the extrameddulary device like dynamic screw in trochanteric fracture of femur. ${ }^{1,2}$ Recent study find out some complication with gamma nail like shaft of femur fracture $^{3}$, fixation failure ${ }^{4}$, distal locking complication need to reoperate but they are very few cases . ${ }^{3,4}$ Gamma nail are still widely used despite of having such complication. The comparative data of gamma nail and PFNA result that PFNA is little better device in unstable proximal fracture. ${ }^{2,5}$ Something about gamma nails which is used in my hospital since last three years. The proximal femoral nail anti rotation (PFNA; Synthes, Oberdorf, Switzerland) was developed by AO/ASIF (Davos, Switzerland) as an alternative to the proximal femoral nail. It has small distal shaft diameter which causes less stress co centration of tip. The PFNA blade compact the cancellous bone and provide stability and prevent retard rotation and varus collapse. Since its introduction, so many clinical studies have shown very good result with very little intraoperative problems and post operative complication. ${ }^{6}$
\end{abstract}

Key words: Intertrochanteric fracture, PFNA (proximal fixation nail antirotaion), Gamma nail.

\section{Material and method:}

All patients with unstable proximal trochantric fracture of my hospital between 2010 Sep to May 2012 was observe for the study .Criteria for Participation in this Clinical Trial Inclusion Criteria:

1- Age 55 years and more. 2- Patients with isolated, unstable, closed or type 1 open trochanteric fractures, classified as AO 31-A2 or AO 31-A3. 3- Definitive primary fracture treatment with PFNA or Gamma 3 within three days after the index event. 4- Signed written informed consent (by the subjects or legal guardian) and agreement to attend the planned follow-ups.

Exclusion Criteria:

1 - Pathologic fracture of any other cause than osteoporosis. 2 - Patients or legal guardian refusing to sign the informed consent form. 3 - Multiple traumas. Type 2 and 3 open fractures. 4 - Drug or alcohol abuse. 5- Wound and/or bone healing disorders of any other cause than diabetes mellitus or smoking. 6 - Active malignancy. 7Expected life expectancy $\leq 3$ months. Inability to walk independently prior to injury event (use of one stick is allowed. Not meeting inclusion criteria are bedridden, wheel-chair ridden and walker dependent subjects). 8Neurological and psychiatric disorders that would preclude reliable assessment (eg. Parkinson disease, Multiple sclerosis, severe depression). 9- Rheumatoid arthritis. 10- Gender Eligibility for this Clinical Trial: BothMinimum 11- Age for this Clinical Trial: 55 Years, Maximum Age for this Clinical Trial: N/A. 12- Are Healthy Volunteers Accepted for this Clinical Trial: Accepts Healthy Volunteers?

Background parameter, ASA [American society of anesthesiologist] risk scores 1 to 4 and preadmission mobility record.

The standard protocol for both Gamma nail and PFNA was followed as earlier mention. ${ }^{7}$ The PNFA which was used for study made by titanium nail with length of 170 or $240 \mathrm{~mm}$ and diameter of 10 to $11 \mathrm{~mm}$ with meddulary nail with mediolateral curvature of 6 degree which is inserted without reaming of 
inetrameddulary canal Operation time, fluoroscopy time, blood loss, and any intraoperative complications were recorded. Standard plain anteroposterior (AP) and lateral radiographs were obtained on the first postoperative day, or during the hospitalization period. The quality of reduction of the fracture was classified as good if alignment was normal, acceptable $\left(5^{\circ}-10^{\circ}\right.$ varus/valgus and/or ante version/retroversion), or poor $\left(>10^{\circ}\right.$ varus/valgus and/or ante version/retroversion). The ideal position of the blade or screw was defined as being central or inferior on the AP radiograph and central on the lateral radiograph.

The PFNA may be distally locked either dynamically or statically. Gama nail have $170 \mathrm{~mm}$ annulated steel nail with lower 4 degree mediolateral curvature and the diameter is of $11 \mathrm{~mm}$. The femur was reamed $2 \mathrm{~mm}$ larger than the proximal and distal diameters of the nail, and insertion was performed manually without hammering. There is 1 distal locking screw for anti rotation. The neck shaft angle of the 2 devices was $130^{\circ}$. The PFNA and the GN-3 were inserted using the percutaneous technique.

First post radiograph was A-P and lateral view and also during hospitalisation.The quality of reduction was taken as good if the alignment was normal, acceptable if varus/valgus/ant version/retroversion. The ideal of blade or screw is central inferior to the on A-P radiograph and can take lateral radiograph also.

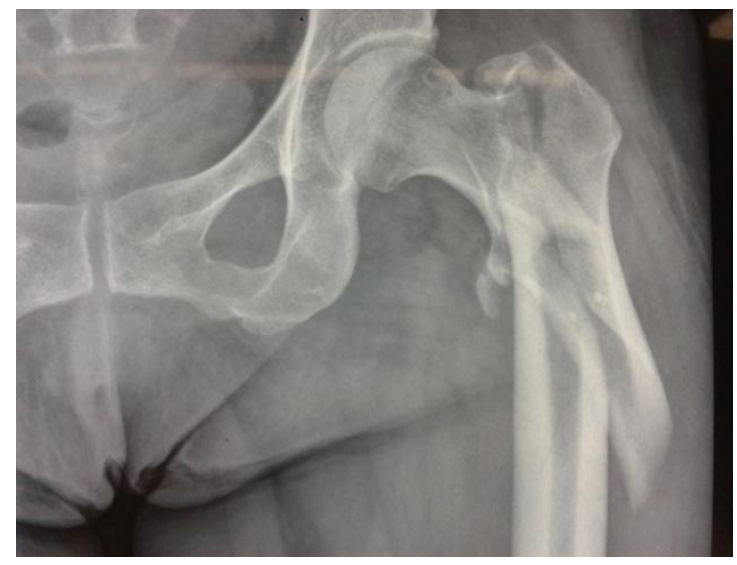

Pre operative radiograph of trochantric fracture $\&$ inta operative radiograph and PFNA
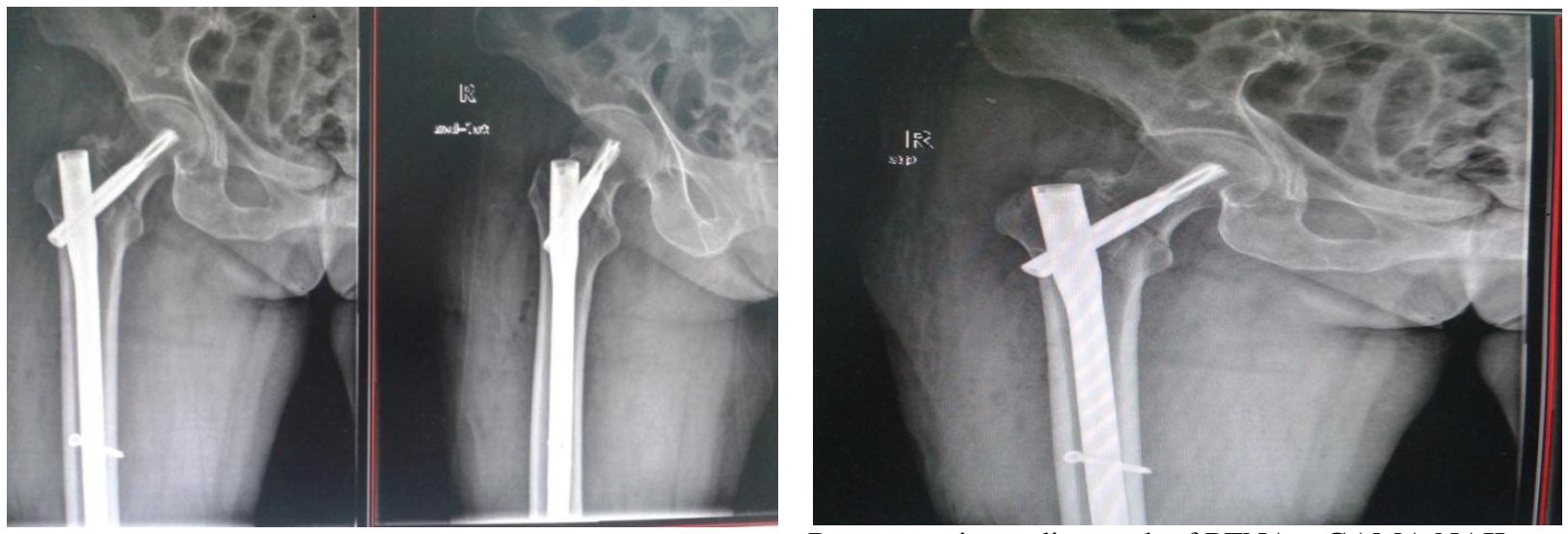

Post operative radio graph of PFNA GAMA NAIL 


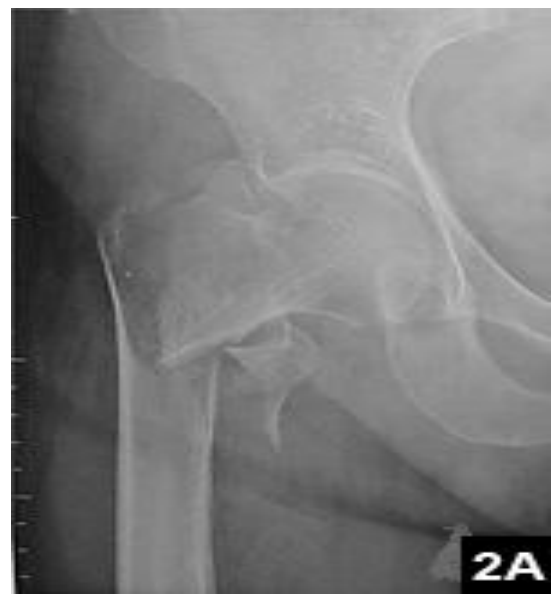

$2 \mathrm{~A}$ - pre operative

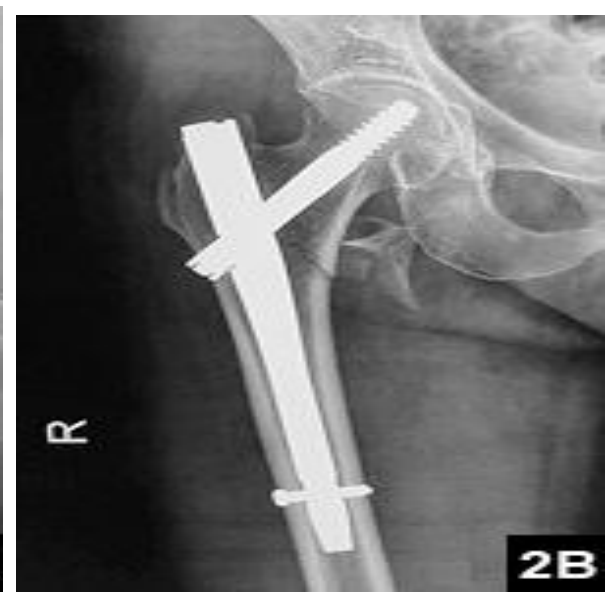

2B-post operative

\section{Result:}

The treatment of the two groups on the basis of injury, age, sex, ASO, mobility before injury. ASO classification fracture pattern in the both group... is given in the following table. The anesthetic agent was same in both cases it was general spinal anesthesia. The fluoroscopy time was longer in Gama nail than PFNA. Fracture reduction was good in the both cases. Time of fracture healing was better in PNFA than Gama nail. The blood loss was less in the PNFA than Gama nail. The implant position was good in most of the cases in both devices on postoperative radiograph. Intra and postoperative complication is given in list 1, 2 and 3.femar shaft fractures are seen intra and post operative in both the condition. There are no cases of non union in both. Complete weight bearing occurs by near one and half month.

\begin{tabular}{|c|c|c|c|}
\hline \multicolumn{4}{|c|}{ Table 1} \\
\hline \multicolumn{4}{|l|}{ Baseline group data } \\
\hline & PFNA & GN-3 & $P$ Value \\
\hline No. patents & 55 & 45 & \\
\hline No. women & 29 & 33 & .7807 \\
\hline Mean patient age, & $75 \pm 1.2$ & $74 \pm 1.0$ & .7049 \\
\hline \multicolumn{4}{|l|}{ ASA score } \\
\hline 1 & 19 & 24 & \\
\hline 2 & 26 & 20 & \\
\hline 3 & 9 & 12 & \\
\hline 4 & 7 & 9 & \\
\hline Fracture type & & & .5696 \\
\hline A2 & 37 & 49 & \\
\hline A3 & 6 & 8 & \\
\hline Mode of injury & & & .7694 \\
\hline Fall at home & 31 & 34 & \\
\hline Fall from height & 7 & 5 & \\
\hline RTC & 10 & 13 & \\
\hline Walking ability score & $7 \pm 0.28$ & $6.8 \pm 0.26$ & .6726 \\
\hline
\end{tabular}

ASA=American association anesthetic

RTC=Road traffic accident

PFNA=Proximal fixation nail anti rotation

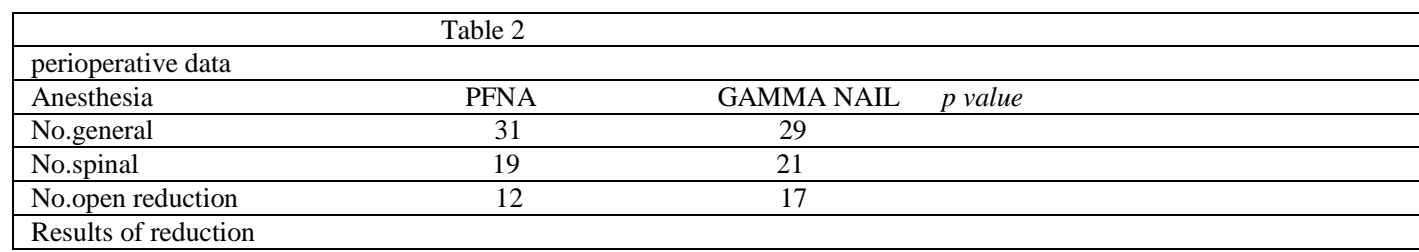


Observational study of proximal fixation nail anti rotation and gamma nail in ...

\begin{tabular}{|lrrr|}
\hline Good & 33 & 37 & \\
\hline Acceptable & 11 & 16 & \\
\hline Poor & 3 & 2 & .1413 \\
\hline Mean operating time,min & $60 \pm 1.8$ & $68 \pm 2.4$ & .0071 \\
\hline Mean blood loss,ml & $217.3 \pm 12.6$ & $272.5 \pm 14.8$ & .0081 \\
\hline Mean fluroscopy time,min & $2.7 \pm 0.14$ & $3.1 \pm 0.13$ & .3467 \\
\hline No. patients transfused & 21 & 29 & .7767 \\
\hline Mean units blood transfused & $1.95 \pm 0.90$ & $2.03 \pm 0.98$ & .5040 \\
\hline Mean hospital stay,days & $7.2 \pm 0.2$ & $7.4 \pm 0.2$ & .1836 \\
\hline Mean time fracture healing,weeks & $9.5 \pm 2.2$ & $10.2 \pm 2.8$ & \\
\hline
\end{tabular}

\begin{tabular}{|lccc|}
\hline \multicolumn{4}{c|}{ Table 3 } \\
\hline complication & \multicolumn{3}{l|}{} \\
\hline & PFNA & GN-3 & $P$ value \\
\hline Femoral shaft fracture & 1 & 1 & .3513 \\
\hline Superficial wound infection & 7 & 7 & .5168 \\
\hline Hematoma & 1 & 2 & .6661 \\
\hline Lateral migration & 3 & 1 & .3151 \\
\hline Decubitus ulcer & 2 & 2 & .7355 \\
\hline Chest infection & 3 & 2 & \\
\hline Urinary tract infection & 6 & 5 & \\
\hline
\end{tabular}

Standard plain anteroposterior (AP) and lateral radiographs were obtained on the first postoperative day, or during the hospitalization period. The quality of reduction of the fracture was classified as good if alignment was normal, acceptable $\left(5^{\circ}-10^{\circ}\right.$ varus/valgus and/or ante version/retroversion), or poor $\left(>10^{\circ}\right.$ varus/valgus and/or ante version/retroversion). The ideal position of the blade or screw was defined as being central or inferior on the AP radiograph and central on the lateral radiograph.

\begin{tabular}{|lccc|}
\hline \multicolumn{4}{|c|}{ Table 4 } \\
\hline \multicolumn{4}{|c|}{ Postoperative Functional Results at Last Follow-up } \\
\hline & PFNA & GN-3 & $P$ value \\
\hline Mean walking ability score & $5.3 \pm 0.29$ & $5.1 \pm 0.19$ & .9147 \\
\hline Mean hip flexion, deg & $98.3 \pm 2.1$ & $94.7 \pm 2.2$ & .2501 \\
\hline No. patients with hip thigh pain & 17 & 9 & .0617 \\
\hline No. of patients recovering walking & 27 & 32 & .6087 \\
\hline ability to preoperative status & & & .7313 \\
\hline Mean femur shortening, mm & $4.3 \pm 0.35$ & $5.0 \pm 0.38$ & \\
\hline
\end{tabular}

III. Discussion:

By observing of these two surgical devices treatment in intertrochanteric fracture I did not found very big difference in the procedure and complication .Its depend on the surgeon to choose the device in the surgery? Still I think PFNA is used commonly in my hospital [from last three years using only PFNA] because the blood loss was less and it was better to use in osteoporotic patients because its anti rotational blade fixed to the head of femur properly.

Anesthetic agent (fentanyl is a synthetic opioid analgesic, musle relaxant was rocuronium, midazolam and propofol also was used) was same in both device surgical procedure. But these two devices are better than the dynamic screw.

Strauss et al have mentioned that fixation of head of femur with helical blade was better than standard sliding screw. Similar biomechanical advantage of the blade over screw was reported by Sommmers et $\mathrm{al},{ }^{8}$ in blade cut out resistance gives better trochanteric fixation.

Shaft fracture at the tip of the nail occurred intra operatively which was treated conservatively with full delayed weight bearing. ${ }^{2,3,4}$ After 6 to 8 weeks patient allowed full weight bearing.

Lateral migration has seen in both implant proximal screw and blade. But it was more with PFNA than Gamma nail. $^{2,9}$ it was because of impaction of fracture.

Pain was most common complication when treating such fracture with intramedullary implant especially in hip and thigh. ${ }^{2,7}$ it was because of mismatch of proximal end of PFNA and proximal femur length (short height of Asians). 
Most of the fracture showed union within 3 months and about more than $60 \%$ of patients achieved same a minimally diminished pre trauma Parker Mobility Score without any correlation to the implant used. We compared this result to the result of retrospective study by Simm ermacher RR et $\mathrm{al}^{9}$ who observed pre operative mobility $56 \%$ to $80 \%$ of patients treated with PFNA. It is same for Gamma nail also so overall result was that more than $60 \%$ patients came back to their pre operative status.

\section{Conclusion:}

PFNA was as good as gamma nail but PFNA was minimally invasive procedure with minimal post operative complication and better device for osteoporotic patient. So both devices are good in the treatment of the intertrochanteric fracture of the femur.

\section{Reference:}

[1]. Menezes DF, Gamulin A, Noesberger B. Is proximal fixation nail suitable for treatment of all trochanteric fracture? Clin Orthop Relat Res. 2005 ; (439):221-227.

[2]. Schipper IB, Steyerberg ED, Castelin RM, et al. Treatment of unstable fracture. Randomized comparison of the gamma nail and proximal femoral. J Bone Joint Surg Br. 2004; 86(1):86-99.

[3]. Kukla C, Heinz T, Gaeblet C, et al. The standard gamma nail: A critical analysis of 1,000 cases. J Trauma .2001; 51(1):77-83.

[4]. Ahrengart L, Tornkvisit H, Fornander P, et al. A randomized study of comparison hip screw and gamma nail in 426 fractures. Clin Orthop Relat Res. $2002 ;(401): 209-222$.

[5]. Al yassari G, Largtaff RJ, Jones JW, et al .The AO/ASIF-Proximal femoral nail treatment of unstable trochanteric femoral fracture. Injury. 2002; 33(5):395-399.

[6]. Simm ernacher RR, Ljung gvist $\mathrm{J}$, Bail H, et al. The new proximal femoral nail in daily practice: Result of a multicentric clinical study. Injury.2008; 39(8):932-939.

[7]. Utrilla AL, Reig JS, Mnoz FM, et al. Trochanteric gamma nail and compression hip screw for trochanteric fracture: A randomized, prospective, comparative study in 210 elderly patients with a new design of the gamma nail. J Orthop Trauma. 2005; 19(4):229-233.

[8]. Somners MB, Roth C, Hall H, et al. A laboratory model to evaluate cut out resistance of implants for peritrochanteric fracture fixation. J Orthop Trauma. 2009; 18(6):361-368.

[9]. Herrera A, Dom Ingo LJ, Martinez A, et al. A comparative study of trochanteric fracture treated with the gamma nail or the proximal femoral nail. Int Orthop. 2002; 26(6):365-369. 